\title{
Creating methods and algorithms of adaptive control in biotechnical complexes of corrective action on human respiratory system
}

\author{
N V Ivakhnoํㅗ ${ }^{\text {, S I Zykin }}{ }^{1}$ \\ ${ }^{1}$ Federal State Budgetary Educational Institution of Higher Education "Tula State \\ University”, Lenina Ave., 92, Tula, Russia, 300012 \\ e-mail: natalia_iv@list.ru
}

\begin{abstract}
The article describes methods of adaptive control of the preset resistance in the respiratory complexes with regard to changes in the human condition based on the results of identifying the respiratory system parameters, as well as on the results of modeling of the respiratory system presented as a combination of airway generations, the last of which ends with alveoli. The presented algorithms lay the basis for building intelligent medical systems involving adaptive corrective action.
\end{abstract}

\section{Introduction.}

Creating adaptive training complexes is a rather complicated theoretical problem $[1,2,3]$. That is due first of all to the complexity and variability of the processed signals. Since the equipment produces a controlling effect, it is necessary to simultaneously diagnose the state of the person's respiratory system, process the results in real time, and adjust the load. The solution to this problem is to create a well-validated methodology and to determine the technical parameters of adaptive-type corrective action equipment having new qualitative characteristics in order to ensure the full diversity of applications in curative, restorative and sports medicine.

For the load on the respiratory muscles to be selected, the patient breathes through the complex, which is operating in various modes (Figure 1).

In the process of breathing, the adjustable resistance in the breathing tube connected to the mouthpiece is used to measure by means of sensors the pressure in the breathing tube $P(t)$, which is then transmitted to the monitoring and control unit through the analog signal processing unit [2].

As a result of analysis of the pressure curve, the input parameters of detection (amplitude of the useful signal $a s$, variance $\sigma^{2}$ and the average noise value $a 0$ ) are adjusted for implementing the signal processing model with the purpose of timely recognition of respiratory activity and ensuring a high degree of synchronization between the person's respiration and the complex $[4,5,6]$. 


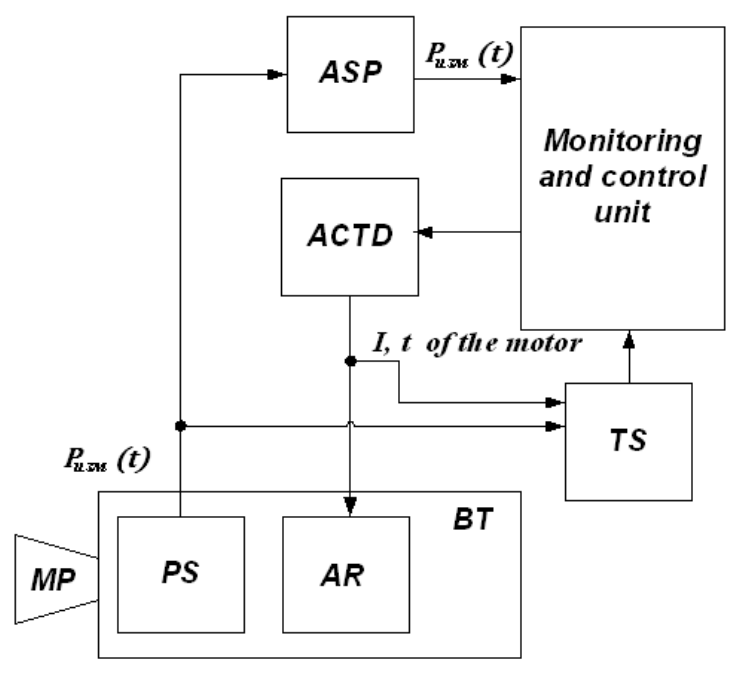

MP - mouthpiece, PS - system of pressure sensors; AR - adjusted resistance preset by changing cross-sectional area; BT - breathing tube;

ASP - analog signal processing unit; ACTD - actuating device; TS - technical self-diagnosis unit

Figure 1. A generalized functional structure diagram for complexes of corrective action on respiratory system.

The respiratory system condition diagnosis unit that employs parametric analysis methods is used to generate control signal sent to the actuating device, which carries out the adjustment and adaptation of the load (resistance/switching pressure) in the breathing circuit according to the predetermined law and taking into account the patient's individual condition and changes in it $[4,5,6,7]$. Also, for the purposes of long-term prediction and for determining limitations on the action, mathematical modeling of processes in the respiratory system is performed.

\section{The criterion used to determine the type of controlling action on the basis of diagnostic matrices.}

In this structure, the self-diagnosing circuit protects the actuating device from overload, analyzing the temperature $t$ and the current $I$ of its electric motor, and allows the failure to be extrapolated by correcting the controlling action. At the same time, the pressure in the breathing tube is analyzed during operation both in the partial load and the peak load mode, which prevents barotrauma $[3,8]$. The anticipatory alert power assessment unit makes it possible to predict a decrease in or failure of the supply voltage level, to save current data in a timely manner, and to ensure the restart and recovery of the system.

Thus, the variable parameters characterizing the state of the person's respiratory system when exposed to different resistances and at different switching pressures $R_{1}, \ldots, R_{N}, P_{1}, \ldots, P_{N}$ are as follows: inspiration / expiration phase duration $T_{1}, \ldots, T_{N}$, slope angle of the approximation curve at the first observation stage $\alpha_{1}, . ., \alpha_{i} . ., \alpha_{N}$, slope angle for the approximation function decrease $\beta_{i}$ (for load in the form of resistance), the coefficient of the approximation function in the third observation interval $\beta p_{1}, \ldots, \beta p_{N}$ (for load in the form of switching pressure), the rise time of the pressure curve to the maximum $t_{H 1}, \ldots, t_{H N}$, characteristics of the pressure curve during free respiration $\alpha_{0}, \beta p_{0}, t_{H 0}, T_{0}$ (load by switching pressure), $\alpha_{0}, \beta_{0}, t_{H 0}, T_{0}$ (load by resistance) $[1,6,7,8]$.

Then, the generalized matrices characterizing the state of the person and the level of their training when exposed to resistance and pressure are described as follows: 


$$
M=\left[\begin{array}{cccc}
\alpha_{0} & \beta_{0} & t_{H 0} & T_{0} \\
\cdot & \cdot & \cdot & \cdot \\
\alpha_{i} & \beta_{i} & t_{H i} & T_{i} \\
\alpha_{N} & \beta_{N} & t_{H N} & T_{N}
\end{array}\right], M 1=\left[\begin{array}{cccc}
\alpha_{0} & \beta p_{0} & t_{H 0} & T_{0} \\
\cdot & \cdot & \cdot & \cdot \\
\alpha_{i} & \beta p_{i} & t_{H i} & T_{i} \\
\alpha_{N} & \beta p_{N} & t_{H N} & T_{N}
\end{array}\right]
$$

The general criterion allowing to establish the type of controlling action is determined on the basis of a set of parameters that make up the matrices of states M and M1:

$$
K 1_{j}^{2}=\left\{\begin{array}{l}
\frac{1}{N} \sum_{k=0}^{s-1} \sum_{i=1}^{N} c_{k} \cdot\left(\frac{M 1_{i k}-M 1_{i}^{*} k}{M 1_{0 k}}\right)^{2} n p u j=1, \\
\frac{1}{N} \sum_{k=0}^{s-1} \sum_{i=1}^{N} c_{k} \cdot\left(\frac{M_{i k}-M_{i k}^{*}}{M_{0 k}}\right)^{2} n p u j=0 .
\end{array}\right.
$$

where $c_{1}, c_{2}, c_{3}, c_{4}$ are weight coefficients characterizing the importance of each indicator, as determined by experts; $j$ is the variable that determines the type of action, $k$ is the parameter number ( $k=0, \ldots, S-1, S$ is the number of parameters, $i$ is the action level $i=1, \ldots, N$, and $i^{*}=i-1$ is the previous action level) $[4,5,9]$.

Then, the task of finding the method of action by load in the breathing circuit in the admissible set of possible options will be reduced to finding such parameters of controlling the actuating device that will provide the best value of the target vector function:

$$
Q_{i}=\left\{\begin{array}{l}
\sqrt{\sum_{k=0}^{S-1} c_{k} \frac{\left(M_{i k}-M Z_{i k}\right)^{2}}{M Z_{i k}^{2}}}, \text { npu } \quad j=0 \\
\sqrt{\sum_{k=0}^{S-1} c_{k} \frac{\left(M 1_{i k}-M Z 1_{i k}\right)^{2}}{M Z 1_{i k}^{2}}}, \text { npu } \quad j=1
\end{array}\right.
$$

where $M Z_{i k}, M Z 1_{i k}$ are the matrices of the required characteristics of the human respiratory system at the given load in the form of resistance and switching pressure, respectively.

Adaptation will be carried out in two circuits: the $1^{\text {st }}$ circuit is responsible for the work of the mathematical model of the respiratory system and the apparatus, the $2^{\text {nd }}$ circuit is responsible for presetting the initial load and for the choice of the value at which the smallest deviation from the reference characteristic is achieved $[1,4,10]$.

\section{The mathematical model of the biotechnical complex "corrective action equipment - human respiratory system".}

For the monitoring and timely adjustment of the load, we will consider a mathematical model of the biotechnical complex "corrective action equipment - human respiratory system" $[5,6]$.

In this model, the structure of the lungs was represented as a set of generations of the airways (channels), the latter of which ends in alveoli. It was assumed that the airways branch out according to the principle of correct dichotomy, that is, two conjugates have the same size and are branched from their parent at equal angles; the empirical equations of the air channels of the average person were taken into account $[6,11,12]$. 
The mathematical model of the biotechnical complex "corrective action equipment - human respiratory system" is represented by three main blocks:

- equations of the gas dynamics of the lungs and of the dynamics of the muscles:

$$
\begin{aligned}
& \int \frac{d P l_{0}(t)}{d t}=\frac{G_{0}(t) \cdot \frac{R b \cdot T^{*}}{M}-P l_{0}(t) \cdot \frac{d V_{0}(t)}{d t}}{V_{0}(t)}, \\
& \frac{d P l_{i m}(t)}{d t}=\frac{G_{i m}(t) \cdot \frac{R b \cdot T^{*}}{M}-P l_{i m}(t) \cdot \frac{d V_{i m}(t)}{d t}}{V_{i m}(t)}, \\
& \frac{d P l_{n m}(t)}{d t}=\frac{G_{n m}(t) \cdot \frac{R b \cdot T}{M}-P l_{n m}(t) \cdot \frac{d V_{n}(t)}{d t}}{V_{n m}(t)}, \\
& \lambda \cdot \rho \cdot \frac{d^{2} u(t)}{d t^{2}}=P l_{n m}(t)-P_{a}-\left(E_{1} \cdot \frac{u(t)}{D c}-\sigma_{1}(t)\right) \cdot \frac{\lambda}{r(t)}-k \cdot \frac{d u(t)}{d t} \cdot \frac{\lambda}{r(t) D c}, \\
& \lambda \cdot \rho \cdot \frac{d^{2} w(t)}{d t^{2}}=P l_{n m}(t)-P_{a}-\left(E_{2} \cdot \frac{w(t)}{L}-\sigma_{2}(t)\right) \cdot \frac{2 r(t) \lambda+\lambda^{2}}{r^{2}(t)}-k \cdot \frac{d w(t)}{d t} \cdot \frac{2 r(t) \lambda+\lambda^{2}}{r^{2}(t) L} ;
\end{aligned}
$$

- equations of medium mass transfer rates by the generation level:

$$
\left\{\begin{aligned}
G_{i}(t)= & \frac{M}{R b \cdot T^{*} \cdot R^{*}{ }_{i m}} \cdot\left[\left(P l_{i m-1}(t)-P l_{i m}(t)\right)^{2} \cdot \operatorname{sign}\left(P l_{i m-1}(t)-P l_{i m}(t)\right)+\right. \\
& \left.+2\left(P l_{i m+1}(t)-P l_{i m}(t)\right)^{2} \cdot \operatorname{sign}\left(P l_{i m+1}(t)-P l_{i m}(t)\right)\right], \\
G_{0}(t)= & \frac{M}{R b \cdot T^{*} \cdot R^{*}{ }_{0}} \cdot\left[\left(P_{k m}(t)-P l_{0}(t)\right)^{2} \cdot \operatorname{sign}\left(P_{k m}(t)-P l_{0}(t)\right)+\right. \\
& \left.+2\left(P l_{1}(t)-P l_{0}(t)\right)^{2} \cdot \operatorname{sign}\left(P l_{1}(t)-P l_{0}(t)\right)\right]
\end{aligned}\right.
$$

where $V_{i m}(t)$ is the current volume of the im-th channel; $n m$ is the last level of the airways $(n m=23) ; R b$ is Boltzmann's constant; $M$ is the molar mass of gas; $\Omega_{i}(t)$ is the volumetric flow rate of gas in the $i$-th channel; $P l_{i m}(t)$ is the pressure of gas in the $i m$-th channel; $P_{k m}(t)$ is the pressure in the tube of the training apparatus; $P_{a}$ is the atmospheric pressure; $P l_{0}(t)$ is the tracheal pressure; $P l_{1}(t)$ is the pressure at the $1^{\text {st }}$ level of the airways (AW); $u(t)$ is the radial displacement of the cylinder wall; $P l_{n m}(t)$ is the current pressure in the cylinder; $\sigma_{1}(t)$ is the preset transverse muscular tension; $\sigma_{2}(t)$ is the preset longitudinal muscular tension; $\lambda$ is the thickness of the cylinder wall; $k$ is the viscosity coefficient of the material; $E_{1}$ is the transverse modulus of elasticity of the material; $E_{2}$ is the longitudinal modulus of elasticity of the material; $r(t)$ is the current inner radius of the cylinder; $D c$ is the inner radius of the cylinder in undeformed state; $\rho$ is the density of 
environment element; $w(t)$ is the longitudinal displacement of the face of the cylinder; $L$ is the length of the undeformed cylinder; $R^{*}{ }_{i m}$ is the resistance of the $i m$-th AW; $G_{i m}(t)$ is the mass air flow rate in the i-th AW; $G_{0}(t)$ is the mass air flow rate in the trachea; the process of respiration occurs at a constant body temperature $T^{*}=310 \mathrm{~K}$;

- the model of automatic switching of respiratory muscles, implemented by means of setting special functions $\Theta_{1}(t), \Theta_{2}(t), \Theta_{3}(t)$ and $\Theta_{4}(t)$, which provide for automatic activation of muscles when the lungs in the process of expiration achieve the minimum preset volume $V_{\min }$ and for disactivation thereof at the moment when the lungs achieve the maximum preset volume $V_{\max }$ :

$$
\begin{gathered}
10^{-5}\left(\frac{d \Theta_{1}(t)}{d t}\right)=\left(1+\Theta_{2}(t)-\Theta_{1}(t)\right) \times \\
\times\left(\varsigma\left(\delta_{V}-V_{\min }-V(t) \mid\right)+\varsigma\left(\delta_{V}-V_{\max }-V(t) \mid\right)\right), \\
10^{-5}\left(\frac{d \Theta_{2}(t)}{d t}\right)=\left(\Theta_{1}(t)-\Theta_{2}(t)\right) \times \\
\times\left(1-\varsigma\left(\delta_{V}-\left|V_{\min }-V(t)\right|\right)-\varsigma\left(\delta_{V}-V_{\max }-V(t) \mid\right)\right), \\
0,5 \frac{d \Theta_{3}(t)}{d t}=\Theta_{4}(t) \cdot\left(1-\Theta_{3}(t)\right)-0,7 \cdot \Theta_{3}(t) \cdot\left(1-\Theta_{4}(t)\right), \\
0,001 \frac{d \Theta_{4}(t)}{d t}=0,5+0,5 \cdot(-1)^{c e i l}\left(\Theta_{1}(t)-\Theta_{4}(t)\right), \\
\varsigma(\arg )=0,5+0,5 \cdot \operatorname{sign}(\arg ),
\end{gathered}
$$

where $\Theta_{1}(t), \Theta_{2}(t), \Theta_{3}(t), \Theta_{4}(t)$ are unknown functions, $\delta_{V}$ is the absolute error of registering the moment when the volumes become equal, ceil is the rounding function, $\varsigma(\arg )$ is the switching function (if the argument is positive, it equals 1 , if the argument is negative, it equals 0 ).

The developed mathematical description of the mass transfer process in the complex system of branching pathways ensures a higher level of detail of the respiration process on the whole, making it possible to study the influence of any changes in the structural level of a specifically selected generation, to the extent of actually assigning given properties to specific airways $[6,7,8]$.

The obtained system of ordinary nonlinear differential equations was solved numerically using Rosenbrock method. An analysis of the experimental graphs of changes in the pressures, volumetric and mass flow rates described in $[6,12]$ shows that the obtained results of modelling provide highly accurate qualitative and quantitative reflection of the biomechanics of a number of processes accompanying respiration. Consequently, this model can be used to analyze the adequacy of and to set limitations on the preset adaptive loads on the respiratory system in various training modes using the CACRS.

A generalized structure diagram of the mathematical model is presented in Figure 2. 


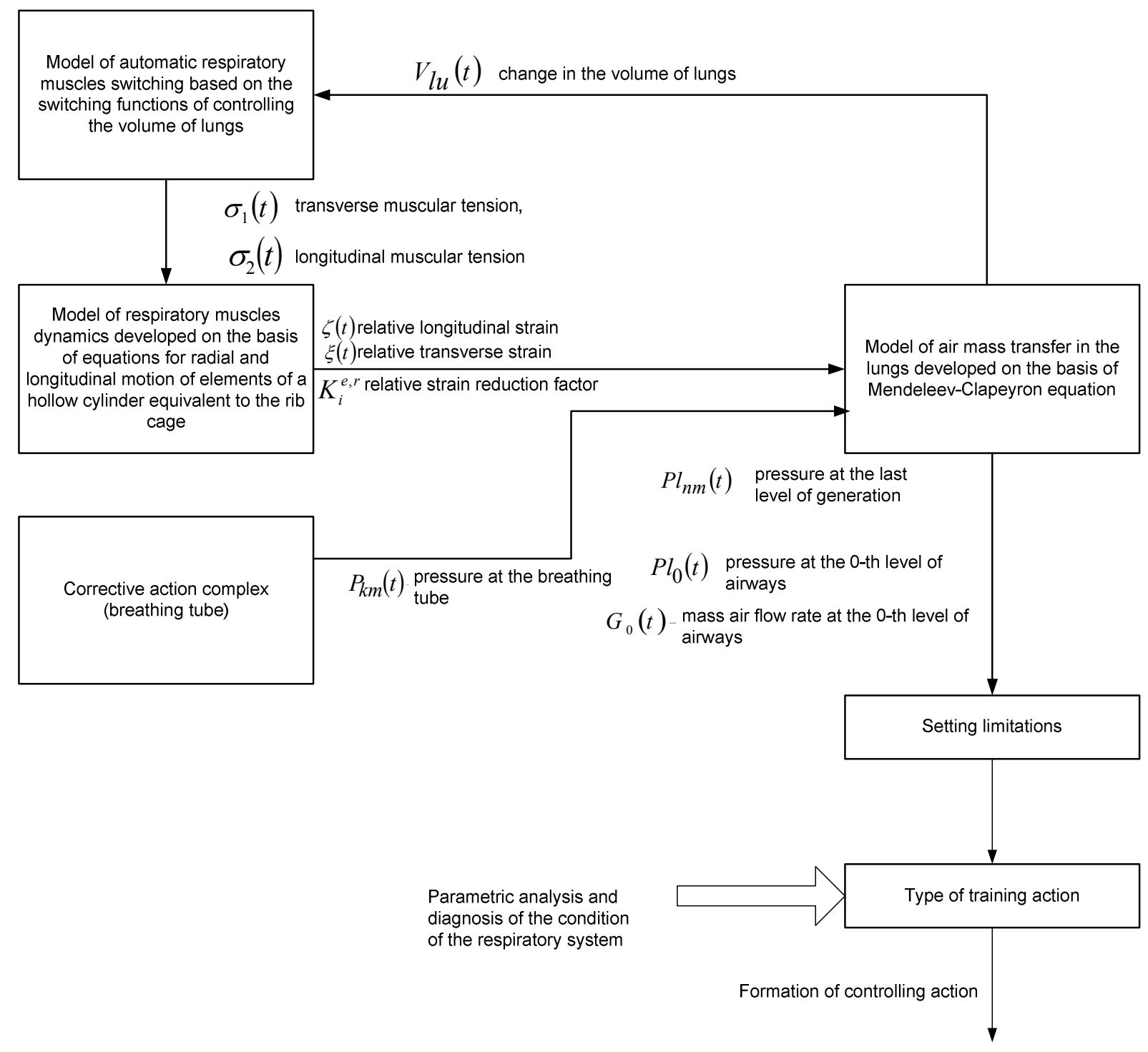

Figure 2. A generalized structure diagram of the model of the biotechnical system "corrective action complex - respiratory system".

\section{Algorithm of load adaptation based on the data of diagnostic condition matrices}

The $2^{\text {nd }}$ circuit operates in search mode with automatic load adjustment, as the parameters of human respiratory system are changing (Figure 3); $N A$ is the number of load alteration levels $[3,11,12]$. 


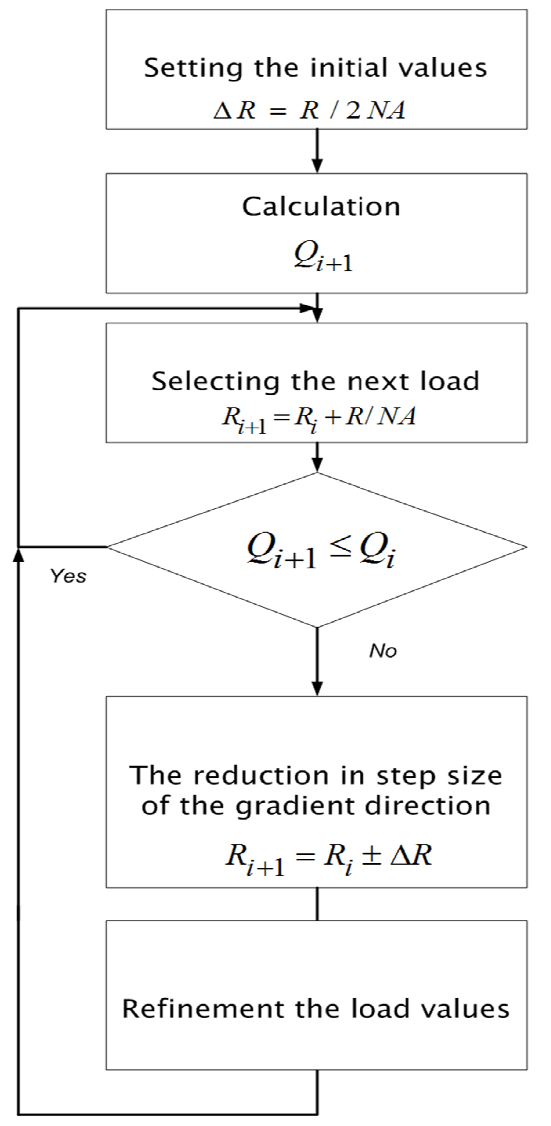

a)

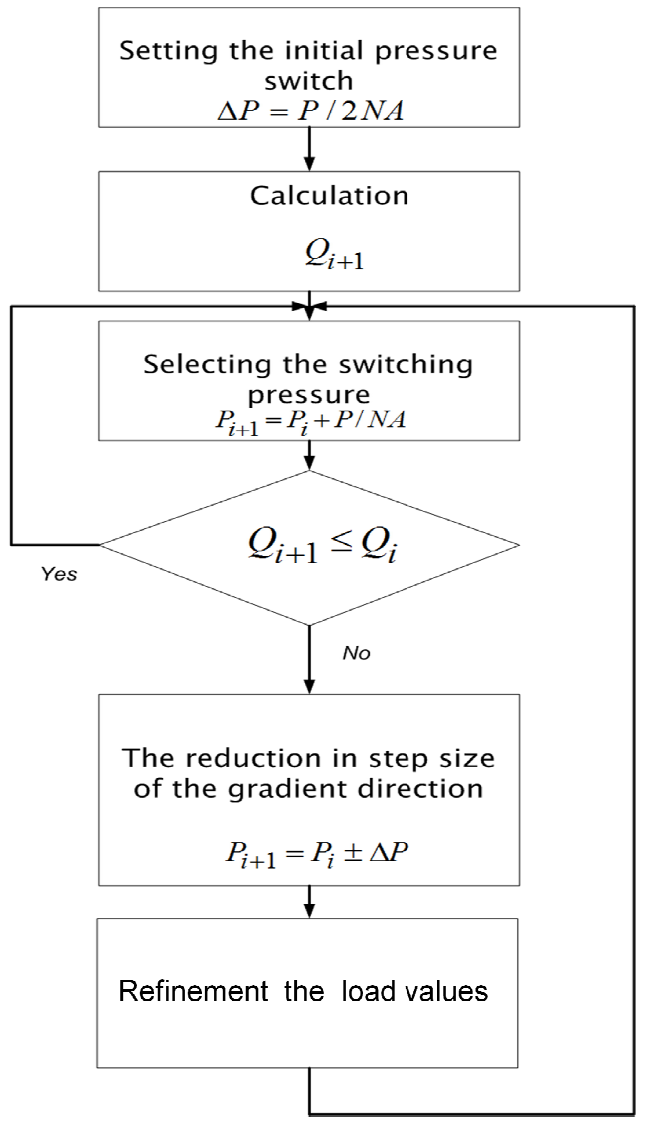

b)

$a$-adjustment of load - resistance of the circuit;

$b$ - switching pressure adjustment

Figure 3. A diagram of work of corrective action complexes adaptation algorithm ( $2^{\text {nd }}$ circuit).

Load correction based on changes in the target function is illustrated by the graph in Figure 4. On the $x$-axis there is every step corresponding to presetting the load for the next inspiration/expiration

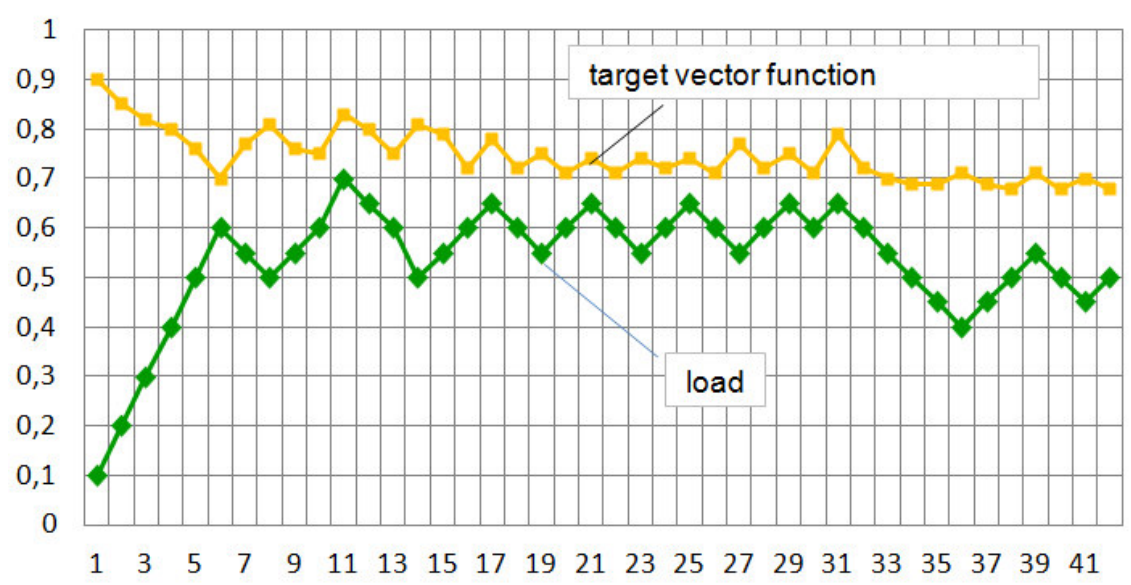

Figure 4. Experimental characteristics of the results of work of the $2^{\text {nd }}$ adaptation circuit (load in the form of resistance). 


\section{Conclusions}

Based on the experimental and analytical assessment of changes in pressure parameters during various types of action on the respiratory system, the problem of automated identification of indicators characterizing the change in the condition of the human respiratory system when working with corrective action complexes has been solved, and a set of algorithms providing timely self-adjustment of the controlling action has been developed.

The task of controlling the actuating device in real time with simultaneous complex signal processing can be accomplished using high-performance microcontrollers.

The above methods and algorithms are the basis for the development of a new class of medical devices: automated complexes of corrective action on the human respiratory system ensuring adaptive signal processing, modeling of biological processes, and real-time control of the actuating device, which increases the efficiency of treating patients with bronchial asthma and chronic obstructive bronchitis, as well as of rehabilitation programs in patients with movement disorders.

\section{References}

[1] Ivakhno N V, Merkulova V 2011 Respiratory muscles simulator Utility model patent No. 115668. Russian Federation. Priority date 20.09.2011

[2] Kravtsova N S, Paringer R A and Kupriyanov A V 2017 Parallel implementation of the informative areas generation method in the spatial spectrum domain Computer Optics 41(4) 585-588 DOI: 10.18287/2412-6179-2017-41-4-585-587

[3] Gaidel A V 2016 Matched polynomial features for the analysis of grayscale biomedical images Computer Optics 40(2) 232-240 DOI: 10.18287/2412-6179-2016-40-2-232-239

[4] Ivakhno N V, Tyagin D 2008 Artificial pulmonary ventilation machine Invention patent No. 2375034. Russian Federation. Priority date 04.06.2008

[5] Ivakhno N V, Fedorov S S 2014 Principle of construction of mathematical model of process of signal processing in the detection of respiratory activity in systems of intellectual fitness effects Biotechnosphere 5(35) 19-22

[6] Ivakhno N V 2015 The structure and algorithm circuit self-diagnosis of the intellectual equipment of respiratory muscles Biotechnosphere 3(39) 40-44

[7] Chafonova V G, Gazeeva I V and Tihomirova G V 2016 Automatic control and digital correction of scale and rotation mismatch in stereo pairs Computer Optics 40(1) 112-121 DOI: 10.18287/2412-6179-2016-40-1-112-120

[8] Ivakhno N V, Antsibor S V 2015 Parametric analysis of respiration characteristics under the relay action Proceedings of the Tula State University. Technical sciences 5(2) 78-84

[9] Gaidel A V, Zelter P M, Kapishnikov A V and Khramov A G 2014 Computed tomography texture analysis capabilities in diagnosing a chronic obstructive pulmonary disease Computer Optics 38(4) 843-851

[10] Ilyasova N Yu, Kupriyanov A V and Paringer R A 2014 Formation of features for improving the quality of medical diagnosis based on discriminant analysis methods Computer Optics 38(4) 851-856

[11] Smelkina N A, Kosarev R N, Nikonorov A V, Bairikov I M, Ryabov K N, Avdeev A V and Kazanskiy N L 2017 Reconstruction of anatomical structures using statistical shape modeling Computer Optics 41(6) 897-904 DOI: 10.18287/2412-6179-2017-41-6-897-904

[12] Antsiperov V E 2016 Automatic target recognition for low-count terahertz images Computer Optics 40(5) 746-751 DOI: 10.18287/2412-6179-2016-40-5-746-751 